\title{
High-Resolution Photoemission Studies of Complex Materials
}

\author{
P.D. Johnson', T. Valla ${ }^{1}$, A. Fedorov' ${ }^{1}$, G. Reisfeld ${ }^{1}$, S.L. Hulbert ${ }^{2}$ \\ 'Physics Department, Brookhaven National Laboratory \\ ${ }^{2}$ National Sychroiron Light Source, Brookhaven National Laboratory
}

\begin{abstract}
Recent instrumentation developments in photoemission are providing new insights into the physics of complex materials. With increased energy and momentum resolution, it has become possible to examine in detail different contributions to the self-energy or inverse lifetime of the photohole created in the photoexcitation process. Employing momentum distribution and energy distribution curves, a detailed study of the optimally doped cuprate, $\mathrm{Bi}_{2} \mathrm{Sr}_{2} \mathrm{CaCu}_{2} \mathrm{O}_{8+\delta}$, shows that the material behaves like a non-Fermi liquid with no evidence for the quasi-particles characteristic of a Fermi liquid.
\end{abstract}

\section{INTRODUCTION}

Combined with the use of synchrotron radiation, photoemission represents one of the premier tools available for the study of the electronic structure of different materials.(1) It has been applied to a variety of systems both in the solid and gas phases. In the early 70 's the technique was extended with the demonstration that by measuring the angle of emission of the photoemitted electrons it was possible to map out the momentum-resolved electronic structure in the solid state.(2) More recently improvements in energy and momentum resolution have allowed a new level of information to be extracted from the data. For example, from detailed investigations of line shapes, several studies have extracted information on the electron-phonon interaction in solids.(3-6) Interaction with the phonons opens a new decay channel for the excitation, resulting in a shorter lifetime and a related mass enhancement. The important point is that here one is looking for effects within a few kT of the Fermi level. The ability to obtain such information has been considerably enhanced with the introduction of new instrumentation.(7) Indeed electron spectrometers are now capable of recording simultaneously the photoemission intensity over a range of energies and angles. We may anticipate that this advance will open up a whole range of new studies. In the present paper we describe a new photoemission facility based on such an instrument and located on the UV floor at the National Synchrotron Light Source. We present several results demonstrating the different capabilities and highlight a study of a high $T_{c}$ superconducting material that clearly shows that it is a non-Fermi liquid. 


\section{THE PHOTOEMISSION PROCESS}

The intensity $I(\underline{k}, \omega)$ measured as a result of the photoemission process is given by:

$$
I(\underline{k}, \omega)=|M|^{2} A(\underline{k}, \omega) f(\omega)
$$

where $\mathrm{M}$ represents the matrix element linking the initial and final states, $A(\underline{k}, \omega)$ is the spectral response and $f(\omega)$ is the Fermi function.(1) The latter enters because the photoemission process represents excitation from the occupied states below the Fermi level. The spectral response $A(k, \omega)$ is given by:

$$
A(k, \omega)=\frac{\operatorname{Im} \Sigma}{(\varepsilon-E-\operatorname{Re} \Sigma)^{2}+(\operatorname{Im} \Sigma)^{2}}
$$

where the imaginary and real components of the self energy $\Sigma$ are reflected in the width of the photoemission peak and its shift from the one-electron Hartree-Fock value respectively. The width of the photoemission peak is, of course, a measure of the inverse lifetime of the excitation. In two-dimensional systems, where final state scattering plays no role, extraction of the self-energy provides us with direct access to the response of the system to the presence of the photohole. This response is manifested in other measurements including transport and optical conductivity.

Determining the imaginary part of the self-energy directly from a traditional photoemission spectrum or energy distribution curve (EDC) can often be complicated by the presence of background emission. Furthermore, if we are interested in the properties of the self-energy in the immediate vicinity of the Fermi level, an added complication is the rapid change in the value of the Fermi distribution function, $f(\omega)$. To obtain an accurate determination of $\operatorname{Im} \Sigma$ it is therefore necessary to deconvolve $f(\omega)$ from the spectrum. This can be achieved by normalizing the data to an experimentally determined Fermi cut-off measured from some material such as gold. Recently Norman et al have proposed another method for extracting the $\operatorname{Im} \Sigma$ from EDCs.(8) Invoking particle-hole symmetry they chose to symmetrise data at $\mathrm{k}_{\mathrm{F}}$ by exploiting the identity $A\left(\varepsilon_{k} \omega\right) f(\omega)+A\left(-\varepsilon_{k_{k}}-\omega\right) f(-\omega)=A\left(\varepsilon_{k} \omega\right)$. However as will be seen, in experimental systems where the intensity is measured in parallel as a function of both energy and momentum, it is often possible and indeed easier, particularly in the vicinity of the Fermi level, to extract the same information from a momentum distribution curve (MDC). In the latter case, rather than measuring the intensity as a function of energy at constant angle, as in an EDC, the intensity is now measured as a function of momentum at a constant energy. The two measurements are simply related. The width of the photoemission peak in the EDC, $\Delta E$, is related to the width of the distribution in the MDC, $\Delta k$, via the band velocity, $v$, such that

$$
\Delta E=2|\operatorname{Im} \Sigma|=v \Delta k
$$


Extracting the information from the MDC minimizes many of the problems associated with the background and the Fermi function cut-off.

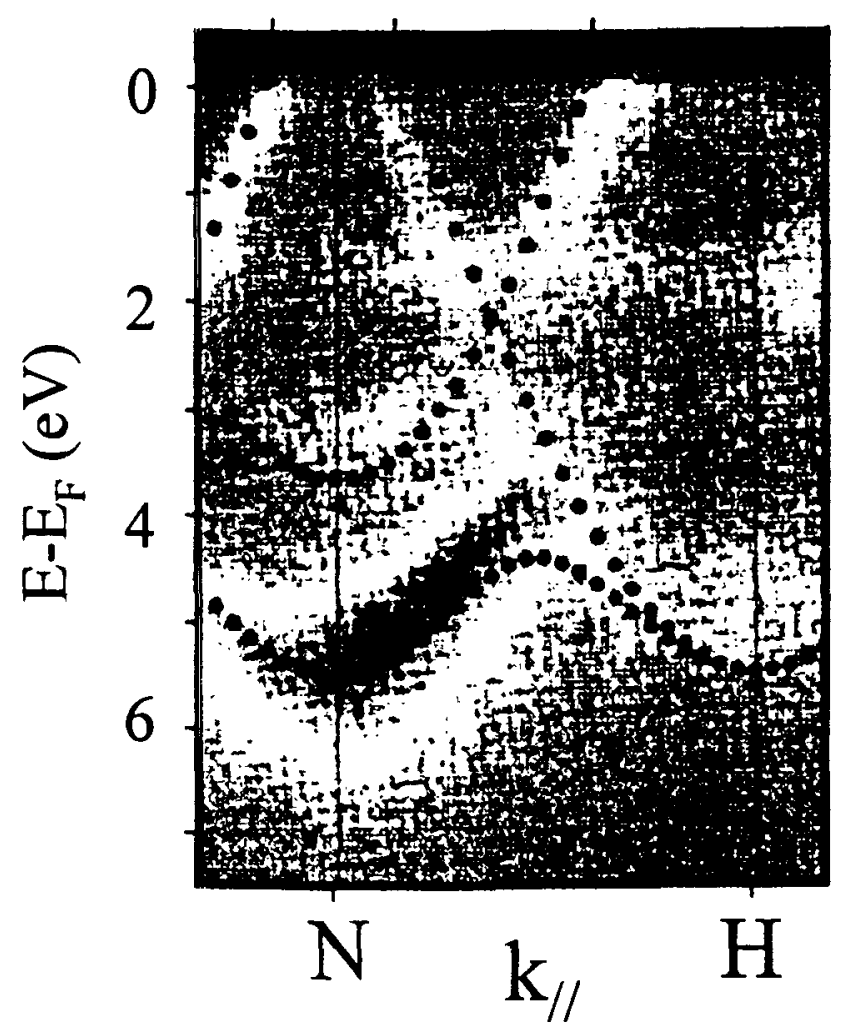

FIGURE 1. Direct observation of the Mo bandstructure along the NH line in the Brillouin zone. The incident photon energy for this study was $390 \mathrm{eV}$. The dotted lines indicate the results of a tight-binding calculation of the bulk bands in this particular direction.

\section{EXPERIMENTAL FACILITY}

The high-resolution photoemission facility at the NSLS consists of an experimental station mounted at the end of a beamline stationed on the U13UV undulator. The monochromator on this line is a Mcphereson $3 \mathrm{~m}$ Normal Incidence device which delivers photons in the range 5 to $30 \mathrm{eV}$ with a resolving power of $>10,000$. The experimental end station, a standard surface-science configuration, is equipped with a Scienta SES200 electron spectrometer capable of operating in the angle-resolved mode. The output of this instrument represents a two dimensional display of the intensity recorded as a function of energy and angle. Driven by software developed at BNL the instrument has the capability of analyzing the 2-d intensity maps to give the traditional EDCs, MDCs as described above, densities as a function of momentum $n(k)$, and intensities as a function of energy at constant $k_{\|}$. The overall energy resolution is of the order 6-10 meV and the angular resolution is of the order of $0.2^{\circ}$. 
The capabilities of the instrument are demonstrated in figures 1 and 2 . The ability of the instrument to multiplex in energy and angle is illustrated in figure 1 where we show the bulk bands of Mo in the NH direction. This image, recorded with incident photons of energy $390 \mathrm{eV}$ derived from the X1B soft $\mathrm{X}$-ray undulator, was obtained in the "fixed mode" such that no voltages are scanned in the analyser during acquisition. In figure 2 we show an MDC corresponding to the Fermi surface crossing of a surface state on the Mo(110) surface.(6) The overall momentum spread including any intrinsic contribution from the surface itself corresponds to $0.36^{\circ}$. It is clear that the actual angular resolution must be les's than this.

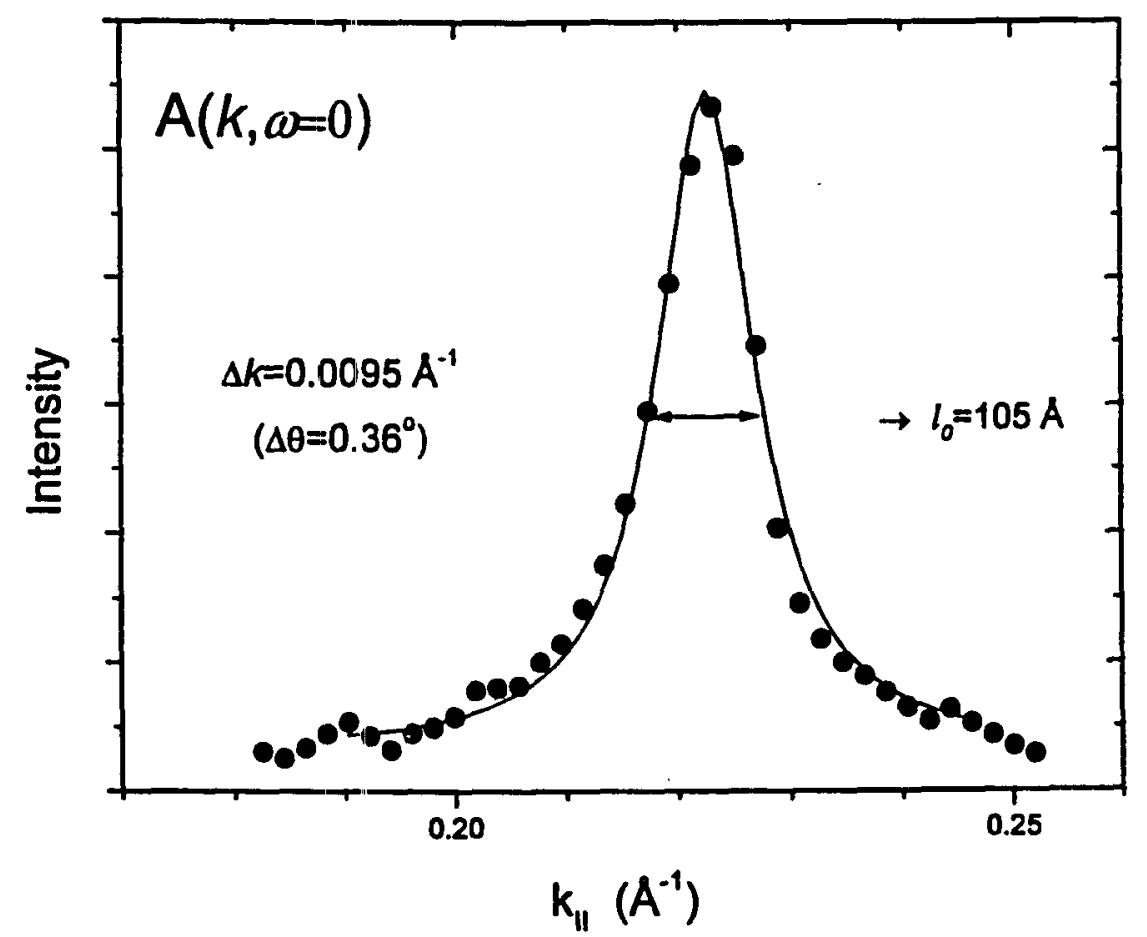

Figure 2. The momentum distribution corresponding to the Fermi surface crossing of a surface state on the (110) surface of Mo. The FWHM of the distribution of $0.36^{\circ}$ corresponds to a momentum spread, $\Delta k$, of $0.0095 \mathrm{~A}^{-1}$. The incident photon energy was $15.16 \mathrm{eV}$.

\section{EXPERIMENTAL RESULTS AND DISCUSSION}

Figure 3 shows a map of the intensity recorded form optimally doped $\mathrm{Bi}_{2} \mathrm{Sr}_{2} \mathrm{CaCu}_{2} \mathrm{O}_{8+\delta}$ along the ( $\left.\pi, \pi\right)$ direction.(9) In this direction there is a node in the superconducting order parameter. In the vicinity of the Fermi level, the intensity sharpens considerably, an observation that is frequently attributed to the presence of well defined quasiparticles. However as we show below, detailed temperature dependent studies as a function of binding energy clearly rule out the presence of quasiparticles as defined for a Fermi liquid.

In figure 4 we compare the two cuts, MDC and EDC, taken from fig. 3 in the vicinity of $\mathrm{k}_{\mathrm{f}}$, the Fermi wavevector. These cuts highlight the two methods of extracting $\operatorname{Im} \Sigma$ as discussed above. In the case of the MDC, we simply take the product of the FWHM of the Lorenztian and the velocity, $v_{\mathrm{F}}$, as determined from the dispersion 


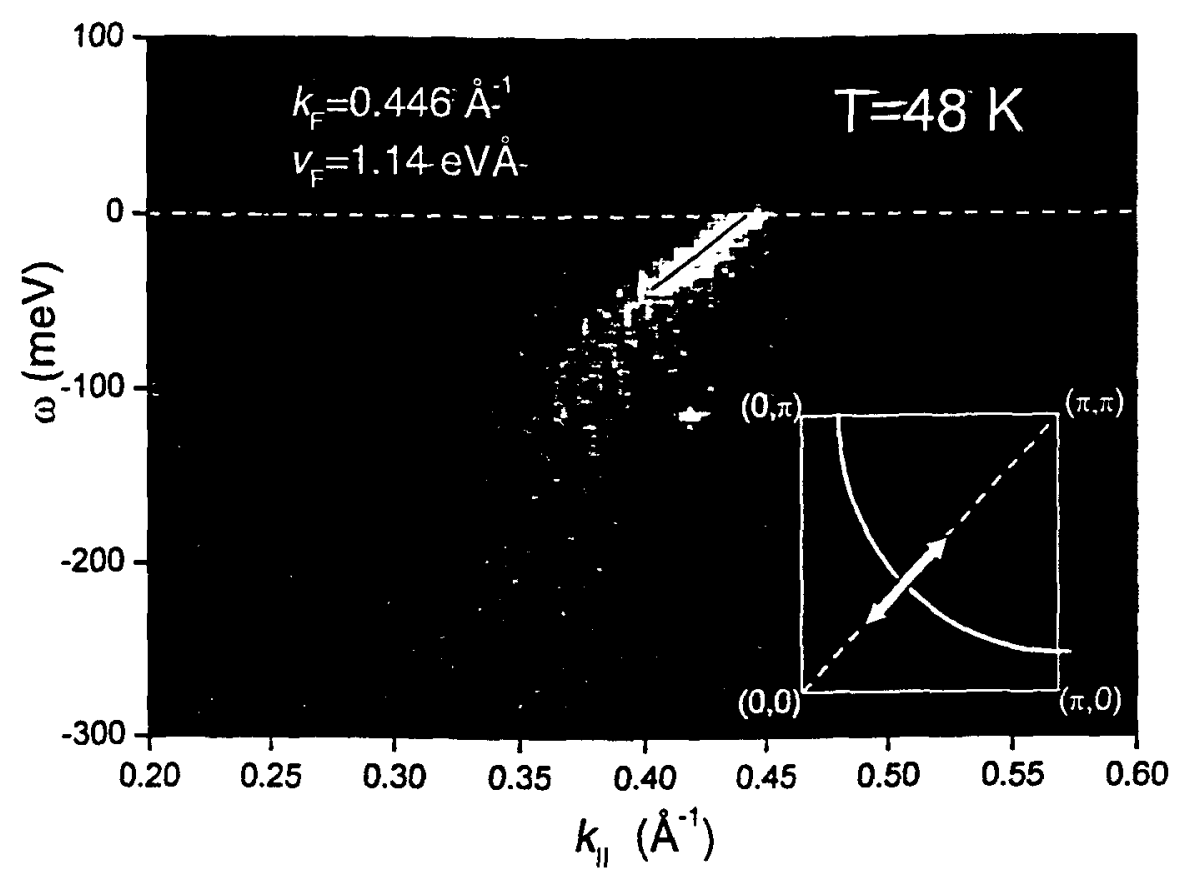

FIGURE 3. Two-dimensional spectral plot showing the intensity of emission in the $(\pi, \pi)$ direction of the Brillouin zone as a function of $\omega$, the binding energy and $k_{\|}$, the parallel momentum. The line in the vicinity of the Fermi level indicates the rate of dispersion used to derive the Fermi velocity. The photon energy is $21.2 \mathrm{eV}$ and the sample ternperature is $48 \mathrm{~K}$. The inset shows the region of the Brillouin zone sampled in the experiment.
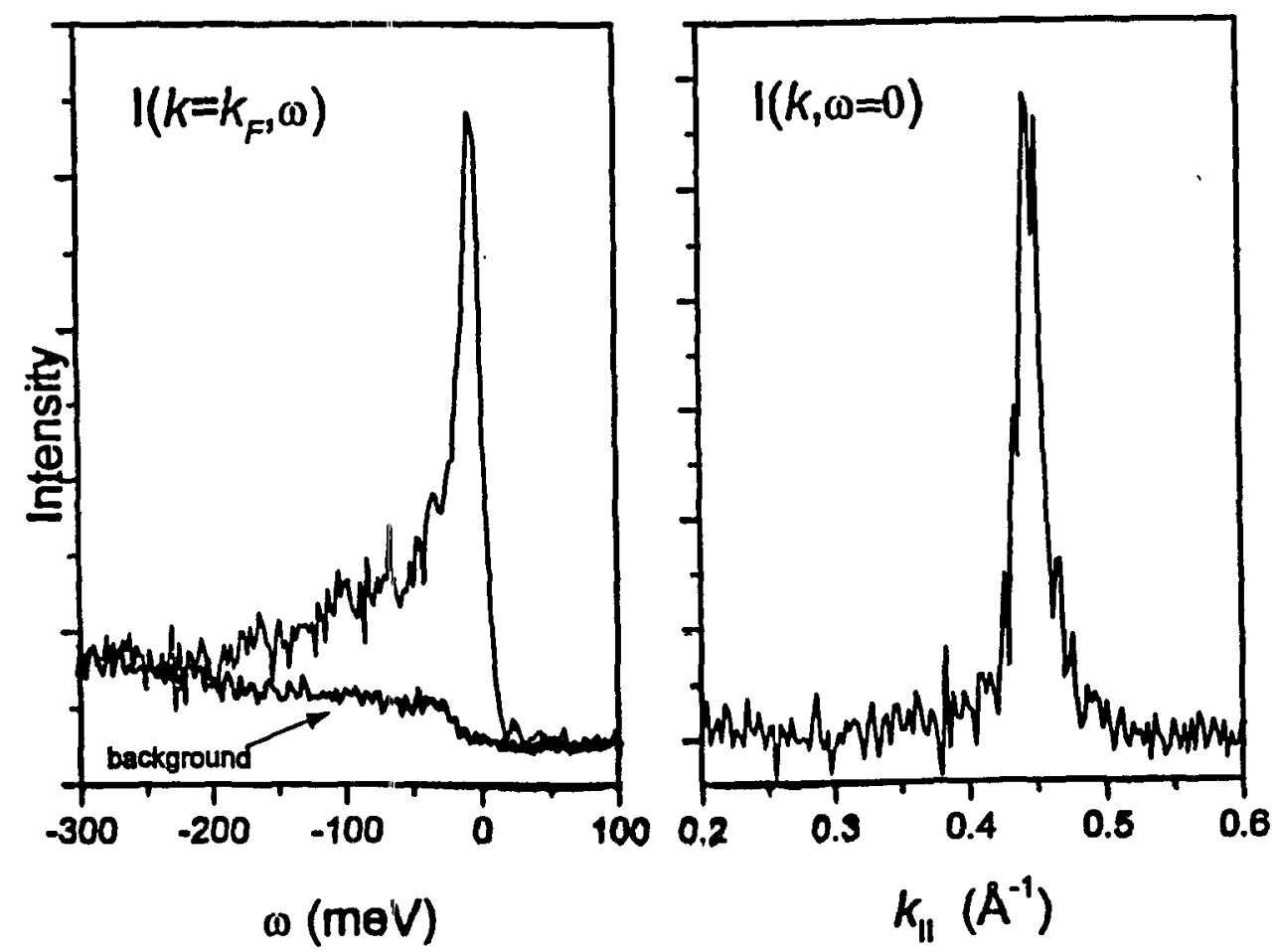

FIGURE 4. (a) An EDC cut through the spectral response plot of figure 3 at an angle corresponding to the Fermi wavevector. (b) A MDC cut through the same data set at a binding energy corresponding to the chemical potential. 
indicated in fig. 3. In the case of the EDC we obtain the same information but now have to take into account the indicated background emission and the effect of the Fermi distribution cut-off. Discussed in detail elsewhere, temperature dependent measurements at $\mathrm{k}_{\mathrm{F}}$ show a linear dependence in the width of the MDC as the temperature is lowered.(9) At higher binding energies away from the Fermi level the width of the peaks in the EDCs are found to scale linearly with binding energy. The crossover from binding energy to temperature dependence scales with the sample temperature as indicated in figure 5 , where we compare $\operatorname{Im} \Sigma$ measured for the high $T_{c}$ material with the same measured from the Mo surface state. For the former the linearity with binding energy and temperature is a clear indication of non-Fermi liquid behavior.(10,11) In a Fermi liquid the self-energy or inverse lifetime reflecting electron-electron scattering would be expected to show a quadratic dependence in both binding energy and temperature.(12) Such a contribution is evident in the Mo data. Another indication of different behavior is obvious in that the width of the photoemission peak is always smaller than the peak binding energy for the Mo data but always larger for the high $T_{c}$ data. The diagonal line in fig. 5 represents the line on which the width of the peak is equal to the peak binding energy.

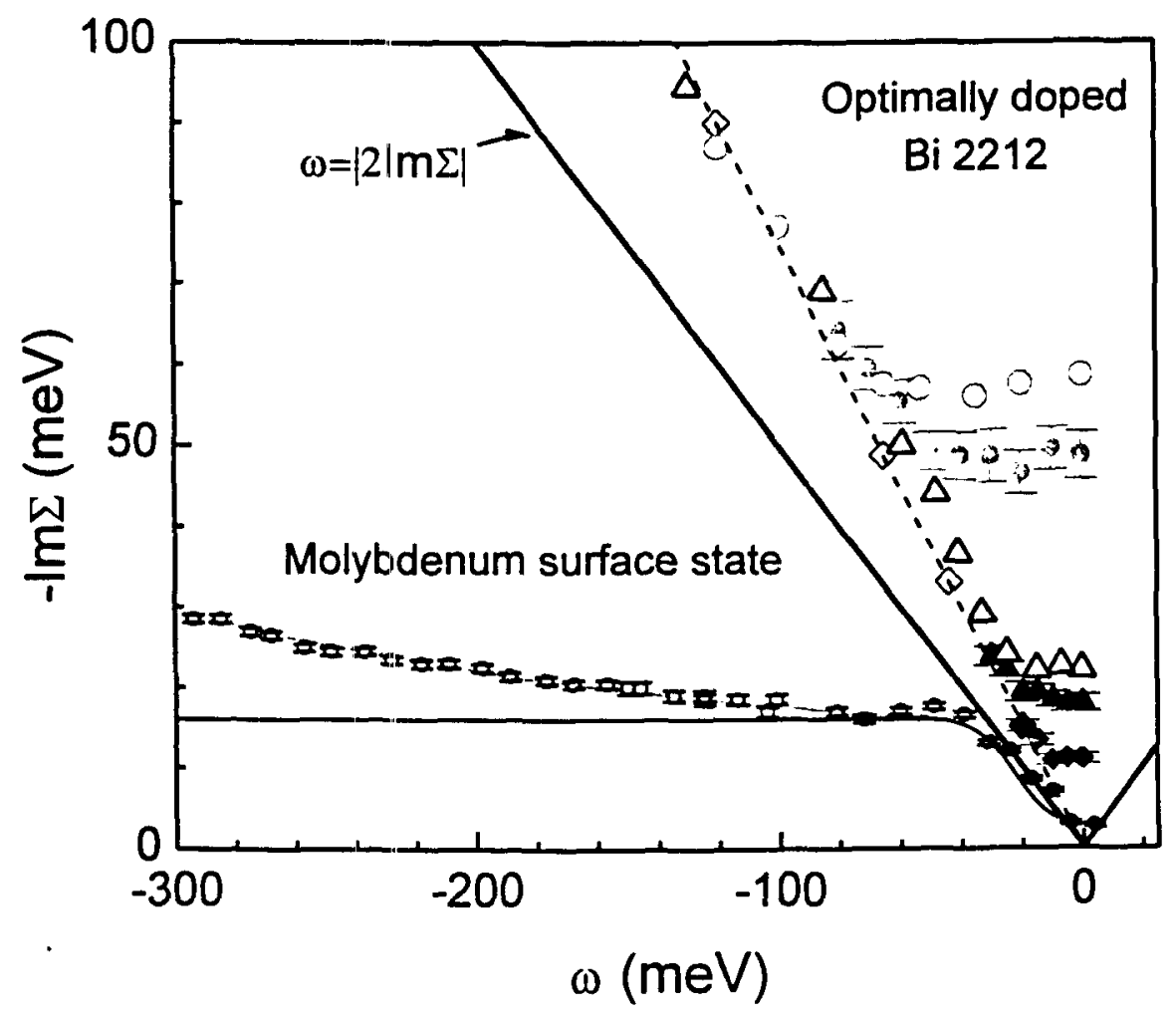

FIGURE 5. A comparison of the innaginary part of the self energy, $\operatorname{Im} \Sigma$, measured for a molybdenum surface state with that measured fo: the high $T_{\mathfrak{c}}$ material. In the latter case the circles, triangles and diamonds refer to measurements at temperatures of $300 \mathrm{~K}, 90 \mathrm{~K}$ and $48 \mathrm{~K}$ respectively. The thin solid line represents a calculation of the electron-phonon contribution to $\operatorname{Im} \Sigma$ for molybdenum. The solid line maps the boundary between the peak width being larger or smaller than the peak binding energy. 


\section{ACKNOWLEDGEMENTS}

The work was supported in part by the Department of Energy under contract number DE-AC02-98CH10886.

\section{REFERENCES}

1. Kevan, S.D., Angle-Resolved Photoemission, Theory and Current Applications, Elsevier, Amsterdam, 1992.

2. Smith N.V., Traum M.M., and Disalvo F.J., Sol.Stat. Commun. 15, 211 (1974).

3. McDougall, B. A., Balasubramianian, T., Jensen, E., Phys. Rev. B 51, 13891 (1995);

Balasubramanian, T., Jensen, L., Wu, X. L., Hulbert, S. L., Phys. Rev B 57, R6866 (1998)

4. Hofmann, P., Cai, Y. Q., Grütter, C., Bilgram, J. H., Phys. Rev. Lett. 81, 1670 (1998).

5. Hengsberger, M., Purdie, D., Segovia, P., Garnier, M., and Baer, Y., Phys. Rev. Lett. 83, 592 (1999)

6. Valla, T., Fedorov, A.V., Johnson, P.D., Hulbert, S.L., Phys. Rev. Lett. 83, 2085 (1999)

7. Scienta SES200 Electron Spectrometer, Gammadata, Uppsala, Sweden.

8. Norman, M.R., et al., Phys. Rev. B 60, 7585 (1999)

9. Valla, T., Fedorov, A.V., Johnson, P.D., Wells, B.O., Hulbert, S.L., Li, Q., Gu, G.D., Koshizuka, N., Science, 285, 2110 (1999)

10. Varma, C.M., Littlewood, P.B., Schmitt-Rink, S., Abrahams, E. and Ruckenstein, A.E., Phys. Rev. Lett. 63, 1936 (1989)

11. Anderson, P.W. in Proceedinys of Fermi International School of Physics, Frontiers and Borderlines in Many-particle Physics (North-Holland Amsterdam, 1987)

12. Pines, D., and Nozieres, P., The Theory of Quantum Liquids (Benjamin, New York, 1969) 